\title{
Problematizing transitions in relation to correctional centres for people living with HIV: Unpacking the taken for granted
}

\author{
Morgan Wadams RN, BScN \\ PhD Candidate, Faculty of Nursing, University of Alberta
}

Cite as: Wadams, M. (2021). Problematizing transitions in relation to correctional centres for people living with HIV: Unpacking the taken for granted. Witness: The Canadian Journal of Critical Nursing Discourse, 3(2), pp 47-63. https://doi.org.10.25071/2291-5796.103

\begin{abstract}
:
Transitions into and out of correctional facilities for people living with HIV are a pivotal point in the HIV treatment cascade where adherence metrics are significantly affected. In this paper I use Alvesson and Sandberg's problematization method of literature analysis to critique and understand the taken-forgranted assumptions underpinning how knowledge is generated within the intersecting fields of HIV, transitions, and corrections. Utilizing problematization, two assumptions underpinning knowledge generation are identified: the linearity of the HIV care continuum model and the tendency to create and perpetuate spatially segregating metaphors of transitions inside versus outside correctional facilities for people living with HIV. These assumptions are discussed in the context of how they shape dominant ways of thinking and practicing in the field. An alternative way to understand transitions for people living with HIV is proposed along with recommendations to guide the HIV care practices of nurses and other healthcare providers.
\end{abstract}

Keywords: HIV, correctional facilities, nursing, problematization, transitions

\section{Introduction}

For people living with HIV (PLWH) and experiencing incarceration or release to their communities, transitions are difficult (Iroh et al., 2018). Although there have been many years of research dedicated to understanding transitions in care around correctional facilities, PLWH continue to face significant challenges accessing essential health and social services, both within and outside of incarcerated settings (Iroh et al., 2018). Nurses occupy a key role in facilitating essential HIV services for PLWH experiencing transitions (Hoang et al., 2016). The purpose of this paper is to rethink the field of research around transitions into and out of correctional facilities for PLWH in Canada. The question to be examined in this paper is if, or to what extent, the current conceptualization of transitions into and out of correctional facilities for PLWH affects ideas around how health practitioners think about and deliver HIV-care services.

\section{Background}

At the end of 2016, there were an estimated 63,110 PLWH in Canada (Government of Canada, 2018), with an incidence of 2,561 new HIV infections in 2018 (Haddad et al., 2019). Incarcerated populations in Canada have a disproportionately higher rate (about ten-times) of HIV than the general Canadian population (Canadian HIV/AIDS Legal Network, 2008). Part of this disproportionate rate stems from lacking access to appropriate harm reduction supplies and services in correctional facilities 
across Canada, such as condoms, safe needle exchanges, safe consumption sites, and tattoo parlors (CATIE, 2018a; Gerster, 2020). These rates are also elevated by an increased prevalence of high-risk sexual behaviors and injection drug use among incarcerated populations (Simonsen et al., 2015). In addition, the interface between living with HIV and the incarcerated landscape is complex, as issues of confidentiality, competing health and social priorities (Kouyoumdjian et al., 2020), and gender-based inequities (Erickson et al., 2019) function within a penal system encouraged to punish rather than rehabilitate (Weinrath, 2016).

In order to address the HIV rate, both in correctional facilities and the general community, Canada has endorsed the 90-90-90 targets set out by The Joint United Nations Programme on HIV/AIDS and the World Health Organization (WHO). The 90-90-90 targets $90 \%$ of people living with HIV know their status, $90 \%$ of those diagnosed are on antiretroviral therapy (ART), and $90 \%$ of those on ART achieve viral suppression - are a representation of the HIV care continuum (or cascade) (Public Health Agency of Canada [PHAC], 2018). The HIV care continuum (see Figure 1) is the 'sentinel image' of the targets and objectives of HIV-care interventions and their associated evaluative criteria, such as CD4 counts, viral loads, linkage to HIV-care services, and ART prescription refills (Mugavero et al., 2013; Woznica et al., 2021). Interventions to deliver HIV care as people transition into and out of correctional facilities predominately follow these clinical outcomes (Iroh et al., 2018). While HIV interventions with these clinical outcomes have accomplished much and continue to have commendable goals, there remain salient issues to PLWH accessing HIV services.

Issues related to accessing HIV services and reintegrating into the community or correctional facility for PLWH are diverse, including stigma, medical mistrust, substance misuse disorders, mental health diagnoses, and tenuous social relations with healthcare providers and other actors (Baillargeon et al., 2010; Kemnitz et al.,
2017; Rozanova et al., 2015; Springer et al., 2011). For healthcare providers, these issues faced by PLWH also pose significant challenges to delivering appropriate HIV care to PLWH in transitory states. When a PLWH transitions into a correctional facility it is often considered an opportunity to diagnose, engage, and treat HIV alongside other social and physical issues impacting HIV care outcomes (Subramanian et al., 2016; Meyer et al., 2014). In the context of the HIV care continuum, transitioning into correctional facilities results in ART adherence and viral suppression rates increasing among individuals, which is often attributed to the structured custodial environment and fewer competing demands (i.e., housing and food security) (Subramanian et al., 2016). However, upon release from correctional facilities, ART retention and viral suppression rates decrease, which is a cause of concern and often attributed to diverse individual and structural barriers (Iroh et al., 2018; Kemnitz et al., 2017; Springer et al., 2011; Swan, 2016).

Transitions into and out of correctional facilities for PLWH are a priority area for further research and knowledge development (Pluznik et al., 2021). While a lot of work has contributed towards developing this field of research, the high incidence rate of new HIV infections, challenges of delivering HIV care to PLWH in transitory states, and longstanding concerns of PLWH accessing services as they transition into and out of correctional facilities suggest we may need to rethink how we conceptualize transitions. Thus, in this paper I utilize a problematization approach to identify the takenfor-granted assumptions underpinning knowledge development within the field and provide an alternative perspective to enhance nursing care in this context.

\section{Method}

Alvesson and Sandberg's $(2011,2013)$ problematization methodology, a critical review method, aims to help researchers generate novel research questions. In this problematization I use a practice of dialectical interrogation to critically examine the intersecting areas of HIV, 
corrections, and transitions research. Problematization is firstly an "endeavor to know how and to what extent it might be possible to think differently, instead of what is already known" (Foucault, 1985, p. 9). Alvesson and Sandberg developed this methodology within their field of management studies in response to traditional 'gap-spotting' approaches of conducting research. Notably, enrolling gapspotting approaches to identify research questions is not an inferior approach compared to problematization, but it may have certain constraints on advancing a field of study beyond its ingrained assumptions. Problematization has also been utilized as of method of critique across a range of complex health and social issues, such as dementia care (Purkis \& Ceci, 2015). Broadly, problematization attempts to reinvigorate a research field that may have become conceptually narrowed, with the endgoal of the methodology being the understanding that "new questions offer starting points for new answers" (Alvesson \& Sandberg, 2011, p. 258). Problematization proposes guiding processes for identifying, articulating, and challenging assumptions: (a) identify a domain of literature, (b) identify and articulate assumptions underlying this domain, (c) evaluate these assumptions, and (d) develop an alternative assumption (or understanding). While the problematization methodology is presented linearly, the process is more iterative and reflexive (Alvesson \& Sandberg, 2013).

\section{Identify Domain: Transitions into and out of Correctional Facilities for PLWH}

Problematization begins by identifying a domain of literature which requires the researcher to conduct a broad search and develop inclusion and exclusion criteria (Alvesson \& Sandberg, 2011). I followed this process by generating a search strategy of a key interdisciplinary database (MEDLINE) with our faculty librarian. ${ }^{1}$ No date limitation was applied. After including 137 texts in the initial broad overview, the conceptual field was narrowed to eight studies. While this reduction in texts is significant, the problematization methodology prioritizes in-depth readings of a few influential texts that represent exemplars of the research or papers that identify important elements of the research problem (Alvesson \& Sandberg, 2011; 2013). The reduction in texts was achieved by including exemplar texts and review papers as well as a bibliography search of these texts to identify and include additional key articles that highlighted the research problem.

\section{Identifying Assumptions}

Alvesson and Sandberg (2011) distinguish five sets of assumptions to both identify and name assumptions after close reading of the texts: inhouse assumptions; root metaphor assumptions; ontological, epistemological, and methodological assumptions; ideology assumptions; and field assumptions. Each assumption, in the order presented, carries with it a broader implication for the field under study. By applying the five different assumptions to the chosen literature, the identification stage tries to transform what are commonly seen as truths or facts into assumptions guiding knowledge generation on transitions of care for PLWH. This process for identifying assumptions was followed by conducting in-depth readings and analysis of eight texts that were produced from the literature search, with a focus on exemplar texts and those that identify important parts of the research problem. Here questions surface, such as: How are transitions for PLWH conceptualized? And, what are the objectives of HIV care during transitions? Two taken-forgranted assumptions informing transitions research around PLWH are identified and described below.

\section{The Linearity of the HIV Care Continuum Model}

A root metaphor assumption, as described by Alvesson and Sandberg (2011), may be recognized by identifying "the basic image or metaphor of social reality informing a text or school of thought" (p. 257). After a close reading of the eight texts, a root metaphor

${ }^{1}$ Please contact the author to obtain a copy of the search strategy. 
assumption informing transitions research for PLWH would be the linear nature of HIV care seen in the HIV care continuum model. The Gardner et al. (2011) text is chosen as a starting point and exemplar work because it initiated and made popular the concept of the HIV care continuum. The HIV care continuum is at the centre of most, if not all, of the included texts that explored, in some way or another, HIV care during transitions around correctional facilities. The image of the continuum begins with those who have HIV, yet are not diagnosed, and ends with those who have achieved viral suppression through ART (see Figure 1). Although there are numerous metaphors and images that influence HIV care during transitions, the care continuum image was prominent across the eight texts. For example, the most recent text included for analysis (Woznica et al., 2021) integrates care continuum targets as a primary outcome in their systematic review.

The linearity of the HIV care continuum model has been challenged as being a largely outcomeoriented and quantitative representation (Mugavero et al., 2013). Alternative models demonstrating the bidirectional and dynamic processes of the continuum have been recommended (Cheever, 2007; Mugavero et al., 2013). However, while these models may influence the types of interventions to enhance diagnosis, engagement in care, and retention in care, they are not the guiding model of an international HIV-care delivery program, like the HIV care continuum. For example, Gardner et al. (2011), who proposed the HIV care continuum model, has $\sim 1900$ citations, whereas the alternative and non-linear model proposed by Cheever (2007) has $~ 170$ citations, and the Mugavero et al. (2013) text, which points to the overly linear nature of the HIV care continuum model, has $\sim 480$ citations. These citation numbers, to me, demonstrate the ingrained uptake of the care continuum in academia and practice. The continuum image attends to the different stages of engagement in HIV care, yet it leaves out precursors or activities that may lead to acquiring HIV, such as intravenous drug use. In addition, this image ends when PLWH achieve viral suppression, neglecting the ongoing sense-making, stigma, and inequities of living with HIV. The HIV care continuum is represented as a linear model with PLWH moving seamlessly from one category onto the next. Interestingly to researchers, practitioners, and policy makers, the care continuum may be linear and static, yet for PLWH proceeding along this continuum, it is dynamic and full of disruption. Gardner et al. (2011) mentioned that PLWH are lost to follow-up, constantly reestablishing care and negotiating care; they also suggested that interventions to achieve HIV-care adherence acknowledge the complexities found between the continuum targets, such as the need for housing assistance. However, the image of the continuum - an identified root metaphor assumption - does not acknowledge the complexities.

\section{Inside and Outside: The Division of a Whole}

After a close reading of the texts, a field assumption encompassing two parts was identified: (a) research tends to conceptualize transitions as those going into correctional facilities and those going out of facilities for PLWH, and (b) we tend to focus on transitions out of correctional facilities. Alvesson and Sandberg $(2011,2013)$ recommend that to identify a field assumption, one must look for a broad idea about a specific subject matter, shared by different schools of thought, and often difficult to recognize because 'everyone' in a paradigm shares it. Various theoretical, quantitative, and qualitative works conceptualized transitions as those going into versus out of facilities and focused on transitions out of correctional facilities for PLWH (e.g., Erickson et al., 2019; Kemnitz et al., 2017; Subramanian et al., 2016). The idea of separating transitions based upon their spatial location is viewed as generally unproblematic and the dominant way of thinking in this field. In addition, the interest and focus of research is mostly on transitions out of correctional facilities, because that is identified as where PLWH re-enter their communities and 'chaotic lifestyles' (Erickson et al., 2019; Subramanian et 
al., 2016, p. 151; Pluznik et al., 2021). This field assumption beckons two questions: Why do we separate transitions, and why do we tend to overly focus on transitions out of correctional facilities?

A common thread that answers both questions is highlighted in Subramanian et al. (2016) and Kemnitz et al. (2017): Most PLWH in the U.S. "receive appropriate HIV care while they are in prison" (p. 1), and ART adherence and viral suppression rates increase when PLWH go into correctional facilities (Iroh et al., 2018). Both insights demonstrate that PLWH usually achieve HIV-related care continuum targets (i.e., CD4 counts, viral load suppression, ART refills), which stand in for 'appropriate' HIV care, while entering and existing within correctional facilities. This reinforces a tendency to create and perpetuate spatially segregated metaphors of transitions going inside versus outside of correctional facilities for PLWH as being heavily influenced by the HIV care continuum's targets. After identifying assumptions in a field of research, Alvesson and Sandberg (2011, 2013) recommend evaluating these ideas in the context of how they affect knowledge generation.

\section{Evaluating the Identified Assumptions}

Merely identifying dominant assumptions does not provide an avenue to pose different questions or lines of thinking. Rather the effects of these dominant lines of thinking need to be challenged and critiqued, so that we can think differently about the problems for PLWH transitioning. Evaluating the identified assumptions encourages further discussion on what 'effects' these assumptions may have on how we come to understand transitions around correctional facilities for PLWH (Alvesson \& Sandberg, 2011, p. 256); specifically, how may these assumptions affect ideas around how healthcare providers think about and deliver HIV-care services? To accomplish this, I assessed the assumptions' effects on knowledge development with creativity and reflexivity to explore how they may have influenced the development of knowledge related to the field (Alvesson \& Sandberg, 2011). Creativity, in this sense, may be understood as creative acts produced by applying diverse methodologies and tools of thought by theorists, disciplines, and individual experiences to the assumptions (Alvesson \& Sandberg, 2011; 2013). Shapin's (1994) and Hacking's (2006) work is utilized as theoretical tools to think alternatively about how the two identified assumptions inform transitions work. Another key question guiding this evaluation was this: How do we come to know or view truth within the field of transitions?

\section{Understanding Effects - The Straight line of the HIV Care Continuum}

Shapin's (1994) argument that knowledge is a social practice, and thus has an ordering effect, may help researchers and practitioners understand how the uptake of the linear HIV care continuum model in research affects what we 'know' about transitions surrounding correctional facilities for PLWH. The generation of knowledge is a collective action but also, a practical activity. Shapin (1994) suggests that knowledge becomes located within people's actions in their everyday lives. This socially constructed knowledge is upheld through trust; without trust collective practices of knowledge generation could not proceed. Shapin states that we place trust in our 'truth-tellers', i.e., those who hold positions where they may contribute to the construction of collective knowledge. For example, it could be assumed that PHAC, WHO, or journals, such as AIDS and Behavior, are regarded as traditional truth-tellers of our society - institutions and entities who speak the 'truth' and may contribute to collectively creating knowledge. In another way, models, such as the HIV care continuum, may be another version of truth-tellers, yet, not as obvious.

The knowledge that the HIV care continuum is based upon, that treatment with ART prevents the transmission of HIV, is something that is trusted amongst HIV care providers (CATIE, 2019). When we utilize this image within transitions work and trust this model, we 
become enrolled in its collective practices of knowledge development. For example, all eight included studies utilized the HIV care continuum targets as primary outcomes. To be involved in these collective practices means that we become a part of the community that 'uses' the HIV care continuum model when conceptualizing transitions into and out of correctional facilities for PLWH and providing HIV care. When we enroll ourselves in this community and the knowledge is utilized and created by those we 'trust', a social ordering effect is then created via the collective actions of actors. To me, this means that the HIV care continuum model may be depicted as a mechanism of social ordering, with both studies and diverse actors seeking to verify, apply, and think along the continuum's measurable targets in HIV research surrounding transitions into and out of correctional facilities. For example, a recent systematic review exploring transitions prioritized HIV care continuum targets (ART refills, viral load suppression, etc.) as key findings that guided their knowledge synthesis and discussion (Woznica et al., 2021). And while it is necessary and commendable to have measurable and generalizable outcomes for HIV care interventions around transitions, these clinical outcomes are often a singular goal of practice in this field that is represented by a linear model. It can also be understood that research syntheses represent the 'state of knowledge' within a given field (SchickMakaroff et al., 2016). As such, I believe an effect may be seen in the way that HIV care is thought about in the minds and practices of those providing HIV care in transitions, such as nurses, so that thinking of HIV care outside of a linear way - which is along the care continuum's targets towards viral suppression may become challenging. Enrolling in the care continuum is important as actors are more closely associated with positions of truth-tellers in our world (Shapin, 1994). When someone does not incorporate the HIV care continuum process when conceptualizing transitions, which is seen as a 'truth-teller', they may be regarded as mistrusting the dominant process. Given that most of the published literature around transitions for PLWH focuses on achieving viral suppression or other care continuum objectives (Iroh et al., 2018; Woznica et al., 2021), I believe it may be challenging for HIV care providers to reconcile their practices that do not move, or have a limited effect on moving, PLWH in transitory states towards these clinical outcomes. Overall, it is important to recognize that endorsing the HIV care continuum can set limits on how we think about care and may constrain what we can know about transitions into and out of correctional facilities for PLWH, as it has social ordering effects on knowledge production and how that knowledge is utilized in practice.

\section{Understanding Effects - Inside and Outside: The Division of a Whole}

From a close reading of the texts, an identified assumption guiding the conceptualization of transitions is the tendency to create and perpetuate spatially segregating metaphors of transitions being inside versus outside of correctional facilities based upon the HIV care continuum's clinical outcomes. Here, HIV care objectives during transitions are in line with the continuum targets, i.e., linkage to care, viral suppression, and CD4 counts (Iroh et al., 2018; Woznica et al., 2021). Hacking's (2006) theoretical work on the social and scientific practices of classification may be utilized to better understand exactly why we tend to create and perpetuate this assumption.

Hacking (2006) describes social and scientific practices of classification as results of the human sciences' (i.e., social sciences, clinical medicine, psychiatry, etc.) attempt to understand, help, control, or encourage certain types of people and their associated ideas. In our pursuit to better understand, help, or act upon one another, our investigations interact with our targets themselves and change them (Hacking, 2006). Two such endeavours to act upon, whether that is to help or control individuals, are the processes of HIV care and incarceration. For example, when someone is diagnosed with HIV, their life drastically changes (PHAC, 2015). Part 
of this change may be the engagement in HIV care. Through the practices of HIV care, a PLWH becomes classified as a certain type of 'object' - a 'person living with HIV'. For incarceration, when someone becomes arrested, charged with a crime, and sent to a correctional facility, they are now another object of inquiry an incarcerated individual. When these two objects of inquiry are merged, such as when someone with HIV becomes incarcerated, they now become an incarcerated PLWH navigating through the judicial system.

The judicial system may be conceptualized and organized as involving two stages, those entering and staying in the system and those exiting. Based upon both social (e.g., an incarcerated individual) and scientific (e.g., person living with HIV) classifications, certain orderings, or ways to classify and organize people, may now apply to a PLWH as they progress through the judicial system (Hacking, 2006). One dominant scientific and social classification, with a prominent ordering effect applied to PLWH, is the HIV care continuum. When the HIV care continuum, as a scientific and social classification with certain evaluative criteria (e.g., CD4 counts, viral counts, ART prescription refills, etc.), meets how the judicial system is organized, two distinct and differing areas of transitions research for PLWH develop: (a) transitions into correctional facilities, where care continuum targets improve, and (b) transitions out of correctional facilities, where care continuum targets decrease.

Care providers and nurses practicing within the field of transitions find their HIV care practices similarly organized - transitions into corrections for PLWH, and thus the care provided, are conceptualized as separate from transitions out of correctional facilities (Erickson et al., 2019). Yet, in the context of individual and structural barriers to accessing HIV services both within and outside of correctional facilities, such as substance misuse disorders, mental health diagnoses, or distrust of medical providers (Baillargeon et al., 2010; Rozanova et al., 2015; Springer et al., 2011), conceptualizing transitions in this manner, and thus HIV care provided, is open to question.

Based upon the ideas of Hacking (2006), the HIV care continuum can be understood as a scientific and social mechanism, categorizing transitions surrounding correctional facilities for PLWH. Within this ordering process, spatially segregating metaphors of inside versus outside for PLWH are created and based upon the evaluative criteria of the HIV care continuum; these are where we see the trajectory of incarceration for PLWH categorized and 'cut'. Thus, in our scientific attempts to better understand PLWH, the dominant ways of thinking - or what we may 'know' and thus act upon (i.e., what is true) - in HIV care practices, is made contingent upon the ordering effects of the evaluative criteria from the HIV care continuum. This is not to say that the ordering effects of the HIV care continuum on transitions is necessarily good or bad, but it has effects; and what has followed from this system of classification is spatially segregating metaphors of inside versus outside which influence how and what types of knowledge are created, taken up by HIV care providers, and thus practiced in HIV care.

\section{Developing Alternative Understandings on Transitions}

An alternative understanding modestly presented here recommends shifting away from the conceptualization of transitions around correctional facilities as a linear process with spatially segregated metaphors of into and out of facilities based upon HIV care continuum targets towards a narrative and Deweyan conceptualization of transitions and experience. To understand transitions composed narratively, I will draw from Clandinin and colleagues (2013) in the discipline of education who explored transitions with youth who left school early. Their work draws on a Deweyan-inspired view of individual experience (Dewey, 1938), which may make the particularities of an individual and their experiences across time visible. In the context of transitions conceptualized narratively, Hacking's (2006) 
work on 'making up people' is presented to illustrate the effects of being enrolled in a system that generates knowledge about an individual, i.e., PLWH, when experiencing transitions.

Clandinin et al.'s (2013) work proposes that instead of understanding transitions as being either into or out of correctional facilities, i.e., a fixed phenomenon with a specific state and position becoming replaced by another fixed state, transitions understood narratively recognize that experiences: shift over time and place; are reflective of lives in a constant process of change; can be understood as liminal spaces; and are shaped by improvisation, imagination, and relationships (Clandinin et al., 2013). From this conceptualization, approaching transitions from a space of liminality would acknowledge that the challenges of delivering and accessing HIV care for PLWH are not divided by a metaphorical 'wall'; instead, they are experienced, albeit to differing degrees, throughout a PLWH's life-course and continuity of experience. For example, consider the challenges of living with a substance misuse disorder by PLWH transitioning around correctional facilities. Within corrections, living with substance misuse is characterized by concerns around tainted drug supplies, overdosing, sharing makeshift rigs (i.e., accessing safe injection equipment), and transmitting sexually transmitted and blood borne illnesses (CATIE, 2018a; CATIE, 2021); these same concerns also characterize the experience of living with substance misuse in the community for PLWH (CATIE, 2018b; CATIE, 2021). If we were to approach transitions as an experience within the theoretical framework proposed by Clandinin et al. (2013), it would begin to attend to both the complexity of transitions for PLWH, and how experiences of transition are not linear nor spatially divided into those going into or out of correctional facilities.

Underpinning Clandinin et al.'s (2013) conceptualization of transitions is a Deweyaninspired view of experience (1938) with its two criteria - interaction and continuity enacted in situations. Understanding individual experience from Dewey's work begins to highlight the complexity of a PLWH's experience in the context of transitions conceptualized narratively. Interaction enacted in situations involves the simultaneous awareness of the internal and external conditions of experience (Dewey, 1938), where one's internal disposition (e.g., an individual's feelings, desires, moral dispositions, beliefs, etc.) is in constant interaction with one's external conditions of experience (e.g., culture, social order/processes, physical environment, etc.). It is the continuous interaction of human thought with our "personal, social, and material environment" that is important (Clandinin \& Rosiek, 2007, p. 5). These interactions are not necessarily in conflict or synergistic with one another, rather they interact and have effects. For example, a PLWH's internal disposition is in constant interaction with their external conditions of experience, such as HIV service providers in a correctional or community setting. Continuity enacted in situations is the understanding that past experiences shape current experiences, while also laying the foundation for a plausible future - "every experience both takes up something from the present moment and carries it into future experience" (Clandinin \& Rosiek, 2007, p. 34).

For example, in the context of living with a substance misuse disorder for PLWH as they transition, continuity enacted in situations may be demonstrated when past experiences of using tainted drug supplies or overdosing lays the foundation for how a PLWH interprets future experiences around accessing harm reduction services.

It is from this understanding of individual experience that we can begin to see that lives do not begin nor end in a correctional setting, and that a Deweyan-inspired understanding of experience begins to highlight these connections between lives, correctional facilities, and outside communities in the context of transitions conceptualized narratively by Clandinin and colleagues. Furthermore, as PLWH transition through correctional facilities and back to the 
community, their lives intersect with those of HIV care providers, namely nurses and other healthcare professionals, and relationships are created. From a Deweyan view of experience, it is understood that HIV care providers are in constant relation with PLWH, as they are not outsiders in the stream of individual experience. As PLWH interact with HIV care providers, these experiences inform future experiences of HIV care. In this sense, HIV care providers participate in both how a PLWH constitutes their experience of transitioning around correctional facilities and how HIV care and carceral systems, within which these care providers operate, affect 'what is known' about PLWH. Hacking's (2006) idea on the concept of 'making up people' highlights the idea presented here of being enrolled - willingly or not - in a system that generates new knowledge about individuals.

\section{Making up People}

The idea of 'making up people' involves the creation, conceptualization, and experiences of a new kind of person through scientific classifications (Hacking, 2006). It is important to note that often this effect occurs because the aim of scientific inquiry is to organize and help in some way (Hacking, 2006). Through trying to organize and help certain kinds of people, such as PLWH, scientific inquiry often enrolls 'engines of discovery', with the goal of uncovering or finding out the 'facts' about certain things (i.e., people, practices, objects). Some of these engines of discovery include counting, quantifying, and normalization, with all engines aiming at producing knowledge and understandings while holding the potential to improve or control 'deviancy' (Hacking, 2006, p. 310). For example: (a) within the field of HIV we produce incidence and prevalence rates (Government of Canada, 2018), i.e., counting as an engine of discovery; (b) we quantify HIV via viral loads, CD4 counts, prescription refills, or HIV specialist appointments attended by PLWH (Subramanian et al., 2016), i.e., quantifying as an engine of discovery; and, (c) we normalize the linearity of how a PLWH should follow the HIV care continuum targets from diagnosis to viral suppression, i.e., normalization as an engine of discovery (PHAC, 2018). Within the context of this paper, the HIV care continuum and its targets are involved in 'making up people', specifically PLWH and how they may be viewed as targets on the continuum's chart (i.e., undiagnosed, engaged in care, non-virally suppressed, etc.) as they transition into and out of correctional facilities. These categorizations are based upon the care continuum objectives (viral load, CD4 count, ART prescription pickups) and inherently a part of how HIV care around correctional transitions is conceptualized (Springer et al., 2011; Woznica et al., 2021). Involved in upholding this system of 'making up people' are HIV care providers.

HIV care providers enroll in their practices around transitions with PLWH the ideas, knowledge, and ways of knowing based upon the HIV care continuum's linearity and spatially segregating metaphors previously discussed. Furthermore, when understood from a position of experience informed by Dewey (1938), i.e., continuity of individual experience, we can also understand that HIV providers shape current and future experiences of transitions for PLWH. As such, the HIV care continuum, and in association the practices of HIV care providers, brings into being certain and very real 'types' of PLWH via Hacking's (2006) engines of discovery - the undiagnosed PLWH, the nonvirally suppressed PLWH, the non-compliant PLWH, or the PLWH engaged and retained in care.

Being categorized as a certain type of PLWH transitioning around correctional facilities has effects on HIV care providers and how a PLWH may internalize their HIV status. For virally suppressed PLWH, care providers may view them as what constitutes a 'good' PLWH; on the contrary, the non-compliant PLWH is often viewed as a deviant or social outcast, posing a danger to society and deserving to be 'fixed'. This poses a question: When we contextualize the linear goals of HIV care around transitions, i.e., move PLWH towards viral suppression, what may occur when PLWH resist these HIV 
care practices? For example, when someone has recently been released from a correctional facility and they are expected to pick up their ART medication, yet they lack housing, food, and employment security (Springer et al., 2011), they may be viewed by nurses as the noncompliant PLWH when they do not show up at the pharmacy or appointments with healthcare providers. If this PLWH is categorized as noncompliant during their next interaction with HIV care, and when we consider that internalized and anticipated stigma towards accessing health services is already high amongst this population (Swan, 2016), how may this affect their future interactions with HIV care providers during transitions? If the HIV care continuum intervenes in both the practices of HIV care providers and what may be known about PLWH and transitions around correctional facilities, how do PLWH make sense of the HIV care providers and numbers that count, quantify, and normalize them? In the context of 'making up' PLWH, we must understand epistemic responsibility as one that acknowledges knowledge making and its application as having consequences (Doucet, 2018).

Approaching transitions and experience from a narrative understanding of transitions and Deweyan-inspired view of experience can begin to highlight the connections between the lives of PLWH, HIV care providers, correctional facilities, and outside communities. When we, by which I mean those whose diverse practices align with HIV care and connect with the lives of PLWH, begin to see these connections, we may then understand that our role in the generation and application of knowledge in HIV care practices is not neutral, but active and having effects. These effects, such as 'making up people', are highlighted when transitions are conceptualized narratively and HIV care providers acknowledge the impact their practices have on how PLWH constitute current and future experiences of transitions and HIV care. It is the hope that this alternative assumption may open avenues for different lines of questioning and ultimately, alternative ways of practicing and knowing in HIV care around transitions.

\section{Discussion: Implications for Nursing Practice}

An alternative way to think about transitions around correctional facilities for PLWH and HIV care is to conceptualize transitions narratively (Clandinin et al., 2013) and approach individual experience from a position informed by Dewey (1938). From this perspective the connections between lives, correctional facilities, and community settings are highlighted, along with the implications of generating and applying knowledge in nursing HIV care practices that are ultimately based upon the linear image and clinical outcomes of the HIV care continuum. Two implications of rethinking transitional HIV care around corrections narratively are discussed: (a) nurses and other HIV care providers may begin to rethink the effects of categorizing PLWH in transitory states around correctional facilities as undiagnosed, non-virally suppressed, noncompliant, or engaged and retained in care; and (b) nurses and others may begin to see the shifts between correctional facilities and communities for PLWH as liminal spaces.

\section{Rethinking Categorizations}

When we view the actions of nurses and other health service providers as participating in what may be known about PLWH in transition, we previously arrived at Hacking's (2006) work on 'making up people' and the relationship between PLWH and care continuum outcomes (viral load, CD4 count, ART prescription pickups). The ideas of the non-compliant PLWH, nonvirally suppressed, or compliant and virally suppressed patient are salient in the minds of HIV care providers - often eliciting diverse images of patients they have provided care to. However, the classification of people - via quantification of HIV outcomes or other means tends to invite stereotypes (Hacking, 2006); a 'good' PLWH is compliant and a 'bad' PLWH is non-compliant. When practitioners deliver HIV care, these stereotypes can - whether consciously or subconsciously - shape how we engage in our conversations and care, with 
negatively viewed or 'bad' patients potentially experiencing further social and structural barriers to HIV care (Geter et al., 2018). In addition, stereotypes may also invite 'evil effects', i.e., the stereotypes experienced by victims become true of themselves - an ultimately self-fulfilling prophecy (Hacking, 2006). Questions must then be raised, such as: How do we truthfully represent the lives of PLWH in HIV care in the context of numbers? And what if these quantified representations of a 'good' or 'bad' patient then become selffulfilling stories of PLWH? How may a nurse work alongside a PLWH who lives with these 'good' or 'bad' representations? While I do not have a direct answer to these questions, thinking about transitions and individual experience narratively can provide a place to begin challenging these potentially negative effects around classifying PLWH in the context of the HIV care continuum and its targets.

HIV care providers also act as a bridge between different worlds for PLWH who have recently experienced incarceration - "the world that do drugs" and "the world that don't do drugs" (Rozanova et al., 2015, p. 8). Being situated in this unique position affords HIV care providers the opportunity to create meaningful relationships with PLWH. Because when HIV care providers see a PLWH's experience in its totality, both in how they adhere to their HIV care and achieve viral suppression but also their lives up until this point in nursing care, they are provided an avenue to begin avoiding interventionist categories of thought applied to PLWH in transitory states. For example, correctional nurses, the largest group of healthcare professionals within correctional facilities in Canada, provide care for people transitioning into facilities while also setting up care trajectories for individuals being released to their communities. They are well-positioned to play a significant role in meeting the healthcare needs of people who are or who have experienced recent incarceration, specifically populations disproportionately affected by HIV and other blood-borne infections (PeterneljTaylor \& Woods, 2019). Being in this position of providing HIV care to PLWH during transitions is a privilege, and alongside privilege comes the responsibility to do right by those whose lives involve a richness that at times during HIV care may be frustrating, but ultimately is rewarding. Understanding transitions conceptualized narratively and as liminal spaces may take this idea further and encourage nurses to make visible the 'wholeness' of a PLWH's life.

\section{Liminality}

Nursing care for PLWH within the justice system involves trajectories of care across correctional facilities and community-based transition programs (Peternelj-Taylor \& Woods, 2019). Part of conceptualizing transitions narratively is seeing transitions characterized as liminal spaces (Clandinin et al., 2013). The word 'limen' means 'threshold,' with a state of liminality being situated upon "uncertain ground, to be leaving one condition or self and entering upon another" (p. 3). Liminality is further characterized by a sense of unsteadiness, where one is not sure where they belong, what they should be doing, or what they even want to do (Heilbrun, 1999). Heilbrun's ideas on liminality resist spatially locating an individual, but instead suggest that a PLWH in a transitory state around corrections is between two worlds the community and correctional facility - while connecting both through their individual experience. If HIV care during transitions is rethought, with the PLWH understood to be in a state of liminality, how may nurses create a space of 'steady' ground when providing HIV care, where the goals and specific lives of PLWH are attended to? To me, when nurses approach HIV care during transitions through a lens of liminality, the wholeness of a PLWH's life is portrayed as well as the barriers to accessing HIV services during transitions.

Barriers to accessing HIV services for PLWH during transitions are diverse, including stigma, medical mistrust, substance misuse disorders, and mental health diagnoses (Baillargeon et al., 2010; Kemnitz et al., 2017; Springer et al., 
2011). As previously discussed, the issues surrounding these barriers, such as living with a substance misuse disorder and concerns around tainted drug supplies (CATIE, 2018a; CATIE, 2021) are not spatially divided by the walls of correctional facilities. When PLWH transitioning around correctional facilities face these barriers to accessing HIV services, how may nursing care providers, coming from an understanding of liminality, support a PLWH when that person is unsure of where they belong, what they should be doing, or what they even want to do? This question is also salient in the context of anticipated and internalized stigma that PLWH experience when accessing health services around transitions (Swan, 2016). A simple, but crucial recommendation presented by Rozanova et al. (2015) is that HIV care providers create relationships with PLWH that forefront respect, understanding, vulnerability, honesty, and a sense of shared decision-making. For most nurses providing any kind of patient care, these insights are not groundbreaking. However, in the context of living with HIV and current and/or past experiences of incarceration, these pillars of a meaningful relationship are inconsistent among care providers (Rozanova et al., 2015).

Overall, the oftentimes chaotic lives of incarcerated and previously incarcerated PLWH do not begin nor end (mostly) in a correctional setting, making it a necessity for nurses and healthcare providers involved in HIV care to understand and attend to the complicated relationships between the incarcerated environment, the PLWH's outside community, and how these intersect with the life of a PLWH. Meaningful relationships with PLWH and HIV care providers should forefront shared decisionmaking and transparency, as well as attend to the barriers of accessing HIV services during transitions around correctional facilities. It is the hope that understanding transitions from a narrative perspective and place of liminality, as well as individual experience from a Deweyaninspired view may serve as a basis to attend to these complicated relations in nursing practice.

\section{Conclusion}

After completing a problematization of the literature on transitions around correctional facilities for PLWH, I identified two taken-forgranted assumptions underpinning knowledge development in the field: the linearity of the HIV care continuum, and the tendency to perpetuate spatially segregating metaphors of transitions inside versus outside of correctional facilities for PLWH. These assumptions affect what we may 'know' and how we practice in nursing and other disciplines by setting boundaries on our understandings to the often linear and clinical outcomes of the HIV care continuum. An alternative approach to this field involves conceptualizing transitions from a narrative understanding informed by Clandinin and colleagues and a Deweyan-inspired view of experience, which begins to highlight the connections between the lives of PLWH, HIV care providers, correctional facilities, and outside communities. When we begin to see these connections, we may then also begin to understand that our role in the generation and application of knowledge in HIV care practices as not neutral, but active and having effects. I believe coming from this narrative perspective of understanding would not go against the dominant, normalizing practices of the HIV care continuum, but instead add a novel dimension of further questioning, and ultimately, add alternative ways of practicing and knowing in the field of transitions around correctional facilities for PLWH.

\section{Declaration of Conflicting Interests}

The author reports no potential conflicts of interest.

\section{Ethical Permissions}

This theoretical discussion required no research ethics board approval.

\section{Funding}

The author is supported in part by a Doctoral Fellowship funded by the Social Sciences and Humanities Research Council.

Data Availability

Please contact the author to obtain a copy of the search strategy. 


\section{References}

Alvesson, M., \& Sandberg, J. (2011). Generating research questions through problematization. Academy of Management Review, 36(2), 247-271. https://www.jstor.org/stable/41318000

Alvesson, M., \& Sandberg, J. (2013). Constructing research questions: Doing interesting research. SAGE.

Baillargeon, J., Hoge, S., \& Penn, J. V. (2010). Addressing the challenge of community reentry among released inmates with serious mental illness. American Journal of Community Psychology, 46(34), 361-375. doi:10.1007/s10464-010-9345-6

Canadian HIV/AIDS Legal Network. (2008). HIV and Hepatitis C in Prisons: The facts. Retrieved from https://www.hivlegalnetwork.ca/site/hiv -and-hepatitis-c-in-prisons/?lang=en

CATIE. (2018a). Harm reduction behind bars: Prison-based needle and syringe programs. Retrieved from https://www.catie.ca/en/pif/spring2015/harm-reduction-behind-barsprison-based-needle-and-syringeprograms

CATIE. (2018b). Harm reduction in action: Supervised consumption services and overdose prevention sites. Retrieved from https://www.catie.ca/en/pif/fall2018/harm-reduction-action-supervisedconsumption-services-and-overdoseprevention-sites

CATIE. (2019). U=U: A guide for service providers. Retrieved from https://www.catie.ca/en/uu-guideservice-providers/very-importantmessage

CATIE. (2021). Safe supply: What is it and what is happening in Canada? Retrieved from https://www.catie.ca/en/pif/spring- 2021/safe-supply-what-it-and-whathappening-canada

Cheever, L. W. (2007). Engaging HIV-infected patients in care: Their lives depend on it. Clinical Infectious Diseases, 44, 15001502. https://doi.org/10.1086/517534

Clandinin, D. J., \& Rosiek, J. (2007). Mapping a landscape of narrative inquiry: Borderland spaces and tensions. In D. J. Clandinin (Ed.), Handbook of narrative inquiry: Mapping a methodology (pp. 35-75). SAGE.

Clandinin, D. J, Steeves, P., \& Caine, V. (2013). Composing lives in transition: A narrative inquiry into the experiences of early school leavers. Emerald.

Dewey, J. (1938). Experience and education. The Macmillan Co.

Doucet, A. (2018). Shorelines, seashells, and seeds: Feminist epistemologies, ecological thinking, and relational ontologies. In Palgrave Handbook of Relational Sociology (pp. 375-391). Springer International Publishing. doi:10.1007/978-3-319-66005-9_19

Erickson, M., Shannon, K., Sernick, A., Ranville, F., Krüsi, A., Pick, N., \& Martin, R. E. (2019).

Women, incarceration and HIV: A systematic review of HIV treatment access, continuity of care and health outcomes across incarceration trajectories. AIDS, 33(1), 101-111. https://doi.org/10.1097/QAD.00 00000000002036

Foucault, M. (1985). The use of pleasure: History of sexuality (vol. 2). Vintage Books.

Gardner, E.M., McLees, M. P., Steiner, J. F., Rio, C.D., \& Burman, W. J. (2011). The spectrum of engagement in HIV care and its relevance to test-and-treat strategies for prevention of HIV infection. Clinical Infectious Diseases, 52(6), 793-800. doi 
https://doi.org/10.1093/cid/ciq2

43

Gerster, J. (2020, March 20). Challenge

to prison needle exchange

program gets its day in court.

Global News.

https://globalnews.ca/news/6629

463/prison-health-addiction/

Geter, A., Herron, A. R., \& Sutton, M.

Y. (2018). HIV-related stigma by healthcare providers in the United States: A systematic review. AIDS Patient Care \& STDs, 32(10), 418-424. https://doi.org/10.1089/apc.2018 .0114

Government of Canada. (2018). Surveillance of $H I V$ and AIDS. Retrieved from https://www.canada.ca/en/publichealth/services/diseases/hivaids/surveillance-hiv-aids.html

Hacking, I. (2006). Kinds of people: Moving targets. Proceedings of the British Academy, 151, 285-318. doi:10.5871/bacad/9780197264249.003. 0010

Haddad, N., Robert, A., Weeks, A., Popovic, N., Siu, W., \& Archibald, C. (2019). HIV in Canada-Surveillance Report, 2018. Canada Communicable Disease Report, 45(12), 304312. https://doi.org/10.14745/ccdr.v4 $\underline{5 \mathrm{i} 12 \mathrm{a} 01}$

Heilbrun, C. G. (1999). Women's lives:

The view from the threshold. University of Toronto Press Incorporated.

Hoang, H. L., Khan, M. N., Berger, S., Moreau, D., Nickel, P., Woods, D., Jaipaul, J., Pyne, D., Moreland, B., Singh, A., \& Ahmed, R. (2016). Assessment of HIV knowledge in correctional facility health care workers: A pilot study of an educational intervention. Journal of the International
Association of Providers of AIDS Care, 15(4), 276-285. https://doi.org/10.1177/2325957 415600797

Iroh, P. A., Mayo, H., \& Nijhawan, A. E. (2018). The HIV care cascade before, during, and after incarceration: A systematic review and data synthesis. American Journal of Public Health, 108(3), e5-e16. https://doi.org/10.2105/AJPH.2015.3026 35

Kemnitz, R., Kuehl, T.C., Hochstatter, K. R., Barker, E., Corey, A., Jacobs., E. A., Repplinger, M. D., Ehlenbach, W. J., Seal, D. W., Sosman, J. M., \& Westergaard, R. P. (2017). Manifestations of HIV stigma and their impact on retention in care for people transitioning from prisons to communities. Health \& Justice, 5(1), 111. https://doi.org/10.1186/s40352-017$\underline{0054-1}$

Kouyoumdjian, F. G., Lamarche, L., McCormack, D., Rowe, J., Kiefer, L., Kroch, A., \& Antoniou, T. (2020). 9090-90 for everyone?: Access to HIV care and treatment for people with HIV who experience imprisonment in Ontario, Canada. AIDS Care, 32(9), 1168-1176. https://doi.org/10.1080/09540121.2019. $\underline{1679710}$

Meyer, J. P., Cepeda, J., Wu, J., Trestman, R. L., Altice, F. L., \& Springer, S. A. (2014). Optimization of human immunodeficiency virus treatment during incarceration: Viral suppression at the prison gate. JAMA Intern Med, 174, 721-29. Doi: 10.1001/jamainternmed.2014.601

Mugavero, M. J., Amico, K. R., Horn, T., \& Thompson, M. A. (2013). The state of engagement in HIV care in the United States: From cascade to continuum to control. Clinical Infectious Diseases, 8, 
1164-1171.

https://doi.org/10.1093/cid/cit420

Peternelj-Taylor, C., \& Woods, P.

(2019). Saskatchewan

provincial correctional nurses:

Roles, responsibilities, and

learning needs. Journal of

Correctional Health Care,

25(2), 177-190.

https://doi.org/10.1177/1078345

819833661

Pluznik, J. A., Nijhawan, A. E., Spaulding, A. C. (2021). Does anything work? Improving HIV care engagement for individuals transitioning out of correctional settings. JAIDS, 86, 286-287.

https://doi.org/10.1097/QAI.000000000 0002599

Public Health Agency of Canada.

(2015). Chapter 3: Populationspecific HIV/AIDS status

report: People living with

HIV/AIDS - living with HIV

and AIDS [webpage]. Retrieved

from

https://www.canada.ca/en/publi

c-health/services/hiv-

aids/publications/population-

specific-hiv-aids-status-

reports/people-living-hiv-

aids/chapter-3-living-with-hiv-

aids.html

Public Health Agency of Canada. (2018).

Summary: Estimates of HIV incidence, prevalence and Canada's progress on meeting the 90-90-90 HIV targets, 2016

[webpage]. Retrieved from

https://www.canada.ca/en/public-

health/services/publications/diseasesconditions/summary-estimates-hivincidence-prevalence-canadas-progress90-90-90.html

Purkis, M. E., \& Ceci, C. (2015).

Problematising care burden

research. Ageing and Society,

35(7), 1410-1428.

http://dx.doi.org/10.1017/S0144

686X14000269
Rozanova, J., Brown, S.-E., Bhushan, A.,

Marcus, R., \& Altice, F. L. (2015).

Effect of social relationships on

antiretroviral medication adherence for people living with HIV and substance use disorders and transitioning from prison. Health and Justice, 3(18), 1-13. https://doi.org/10.1186/s40352-0150030-6

Schick-Makaroff, K., MacDonald, M., Plummer, M., Burgess, J., \& Neander, W. (2016). What synthesis methodology Should I use? A review and analysis of approaches to research synthesis. AIMS Public Health, $3(1), 172-215$. https://doi.org/10.3934/publiche alth.2016.1.172

Simonsen, K., Shaikh, R., Earley, M., Foxall, M., Boyle, C., Islam, K. M., Younger, H., Sandkovsky, U., Berthold, E., \& Margalit, R. (2015). Rapid HIV screening in an urban jail: How testing at exit with linkage to community care can address perceived barriers. Journal of Primary Prevention, 36(6), 427-432. https://doi.org/10.1007/s10935015-0408-1

Shapin, S. (1994). A social history of truth: Civility and science in seventeenthcentury England. University of Chicago Press.

Springer, S. A., Spaulding, A. C., Meyer, J. P. \& Altice, F. L. (2011). Public health implications for adequate transitional care for HIV-infected prisoners: Five essential components. Clinical Infectious Diseases, 53(5), 469-479. https://doi.org/10.1093/cid/cir645

Subramanian, Y., Khan, M.N., Berger, S., Foisy, M., Singh, A., Woods, D., Pyne, D., \& Ahmed, R. (2016). HIV outcomes at a Canadian remand centre. International Journal of Prisoner Health, 12(3), 145156. https://doi.org/10.1108/IJPH-122015-0041 
Swan, H. A. (2016). Qualitative examination of stigma among formerly incarcerated adults living with HIV. Sage Open, 6(1), $1-9$.

https://doi.org/10.1177/2158244016629 524

Weinrath, M. (2016). Behind the walls: Inmates and correctional officers on the state of Canadian prisons. UBC Press.
Woznica, D. M., Fernando, N. B., Bonomo, E. J., Owczarzak, J., Zack, B., \& Hoffmann, C. J. (2021). Interventions to improve HIV care continuum outcomes among individuals released from prison or jail: Systematic literature review. JAIDS, 86(3), 271-285.

https://doi.org/10.1097/QAI.000 $\underline{0000000002523}$

Received May $17^{\text {th }}, 2021$

Revised Sept 27 $7^{\text {th }}, 2021$

Accepted Oct $1^{\text {st }}, 2021$ 


\section{Figure 1}

Canada's 90-90-90 HIV Targets

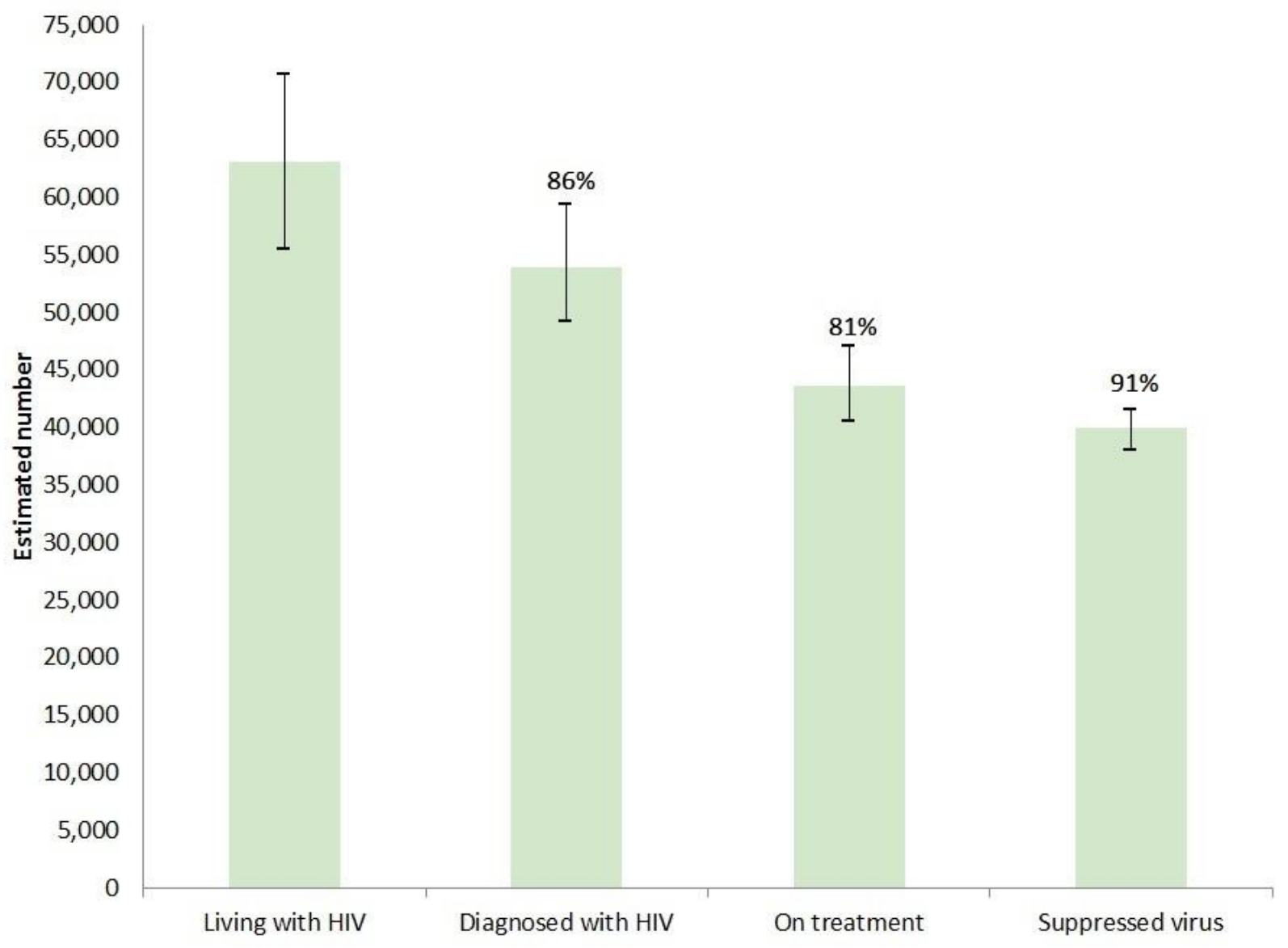

Note. An example of Gardner et al.'s (2011) HIV care continuum. From Public Health Agency of Canada (2018), Summary: Estimates of HIV incidence, prevalence and Canada's progress on meeting the 90-9090 HIV targets, 2016. 